EPJ Web of Conferences 21, 07001 (2012)

DOI: $10.1051 /$ epjconf/20122107001

(C) Owned by the authors, published by EDP Sciences, 2012

\title{
Spin-orbit force contribution to dissipative dynamics
}

\author{
Yoritaka Iwata \\ GSI Helmholtzzentrum für Schwerionenforschung, Planckstrasse 1, Darmstadt, Germany
}

\begin{abstract}
A procedure of obtaining nucleus-nucleus potentials from time-dependent density functional calculations is presented. The role of the spin-orbit force in heavy-ion collision dynamics is studied with respect to the nucleus-nucleus potential. Consequently a novel dissipation mechanism, which should not be classified into the wall-window type dissipation, is proposed.
\end{abstract}

\section{Introduction}

The spin-orbit force has been known to play a prominent role in the nuclear structure [1]. On the other hand a few things have been known for its contribution of to heavy-ion collision dynamics; the introduction of the spin-orbit force was shown to eliminate a part of non-physical fusion window [2]. The fusion window, which was calculated in simplified time-dependent mean-field calculations, is a passing through of colliding nuclei even in collisions at sufficient low energies. For a recent work treating the dynamical dissipation due to the spin-orbit and tensor forces, see [3]. Note that, as is shown in the following, the introduction of the spin-orbit force to the time-dependent mean-field calculations only in two-dimensional axial symmetric framework is not sufficient.

At first a method of obtaining nucleus-nucleus potentials is presented. Next the role of the spinorbit force in heavy-ion collision dynamics is investigated using the obtained nucleus-nucleus potential. Consequently a novel dissipation mechanism, which is not classified into the wall-window type dissipation mechanism [4], is proposed.

\section{Derivation of collective Lagrangian}

\subsection{Collective Lagrangian}

The collective Lagrangian is derived from three-dimensional time-dependent mean-field calculations. Central collisions between the two identical nuclei are concerned, where $z$ is taken to be the collision axis, and the central point of initial positions are set to the origin. This choice of central collisions between two identical nuclei is to prevent introducing any intentional separations between colliding nuclei even after the touching. We introduce the collective variable:

$$
R(t)=2 \iiint|z| \rho(t, x, y, z) d x d y d z
$$

to characterize the relative distance of the two fragments, where $\rho(x, y, z)$ is the density, and $z$ means the direction of the collision axis. We begin with a traditional way of thinking this problem [5-8]. The principle of least action leads to the Euler-Lagrange equation:

$$
\frac{d}{d t} \frac{\partial \mathcal{L}}{\partial \dot{R}}-\frac{\partial \mathcal{L}}{\partial R}=F_{Q} ; \quad \mathcal{L}=\frac{1}{2} \mu(R) \dot{R}^{2}-V(R, \dot{R}),
$$

where $\mathcal{L}$ stands for the Lagrangian, $V(R, \dot{R})$ denotes the nucleus-nucleus potential, and $F_{Q}$ is the nonconservative force (e.g., friction force). $F_{Q}$ is impossible to be described by the potential form. $V(R, \dot{R})$

This is an Open Access article distributed under the terms of the Creative Commons Attribution-Noncommercial License 3.0, which permits unrestricted use, distribution, and reproduction in any noncommercial medium, provided the original work is properly cited. 


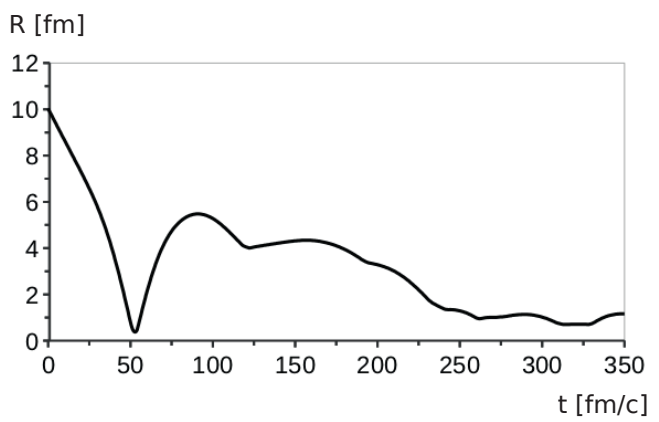

Fig. 1. Time evolution of the relative distance $R(t)$ in a central collision of ${ }^{16} \mathrm{O}+{ }^{16} \mathrm{O}$ at $E_{c . m}=65 \mathrm{MeV}$, where SLy4d parameter set is adopted. This time evolution should be compared to Fig. 1 of [2] in which mean-field and beyond the mean-field calculations (including two-body collisions) are compared in the two-dimensional axial symmetric framework. For precise comparison, the impact parameter, the initial energy, the initial distance, and the sort of colliding nuclei are exactly the same as [2].

is simply denoted by $V(R)$, because we discuss the collision at one fixed incident energy. Note that $F_{Q}$ is equal to zero for $R(t)$ derived from the microscopic dynamics strictly satisfying the adiabatic property (dynamics within the thermal equilibrium), but the inverse statement is not necessarily true. On the other hand the dynamics described by time-dependent mean-field calculations has been confirmed to be far from the thermal equilibrium [9]. For instance the relative distance $R(t)$ (calculated based on the time-dependent density functional calculation) for ${ }^{16} \mathrm{O}+{ }^{16} \mathrm{O}$ is shown in Fig. 1.

The problem here is to determine all the components of macroscopic Lagrangian $\mathcal{L}$ and the nonconservative force $F_{Q}$, which is satisfied by the microscopically determined variable $R(t)$.

$$
\mu \ddot{R}(t)+\frac{1}{2} \frac{d \mu}{d R} \dot{R}(t)^{2}+\frac{d V}{d R}=F_{Q},
$$

where $\mu$ denotes the effective mass. This problem is equivalent to making a relation between microscopic and macroscopic dynamics. We take into account the $R$-dependence of effective mass, where the variance of effective mass during heavy-ion reactions is not understood well.

\subsection{Scheme for determine the Collective Lagrangian}

The unknown components of the collective Lagrangian are deduced step by step. A sketch of the scheme is presented here (the entire process is summarized in [10] based on the traditional manner $[5-8])$. First calculate following two quantities:

$$
V(R(t))=E-\frac{1}{2} \bar{\mu} \dot{R}(t)^{2}
$$

and

$$
V(R(t))-V\left(R_{0}\right)=-\bar{\mu} \int_{t_{0}}^{t} \ddot{R}(\tau) \dot{R}(\tau) d \tau,
$$

where $E$, which denotes the total energy, $\bar{\mu}$ is a constant (reduced mass of the system), $R_{0}$ means the initial distance for the two nuclei, and $V\left(R_{0}\right)=e^{2} Z^{2} / R_{0}$. The former equation is obtained by the conservation of energy, and the latter one follows from the conservation of momentum. Roughly speaking Eq. (3) corresponds to the loss of kinetic energy, while Eq. (4) to all the effects obtained by assuming the potential form. Second compare the two quantities obtained by Eqs. (3) and (4), and classify the calculated values into two different types depending on whether the two quantities are equal or not. If the two quantities are equal for a certain interval of $R$, the corresponding contributions from both $F_{Q}$ and variable $\mu$ is equal to zero. In this case the nucleus-nucleus potential is obtained; the curve obtained by Eq. (3) corresponds to the potential. Third, if the two quantities are not equal for an interval of $R$, the effect of variable effective mass and the contribution from $F_{Q}$ in the corresponding interval are evaluated by the difference between Eqs. (3) and (4). 

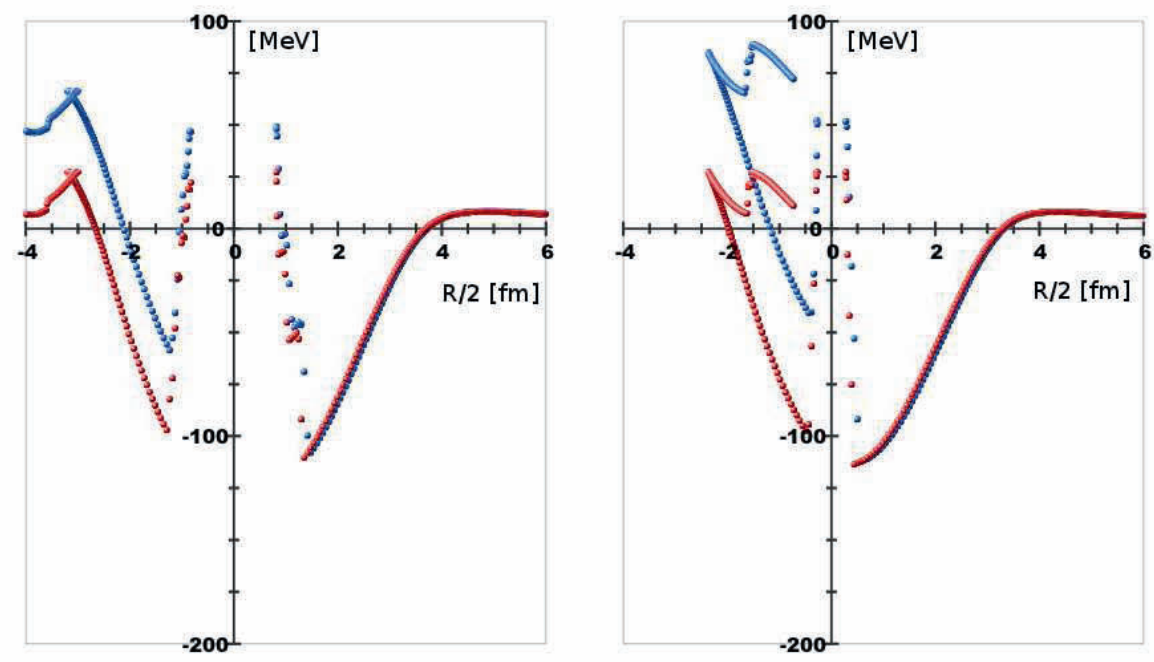

Fig. 2. Time evolutionary plot of the two values (calculated by Eqs. (3) and (4)) for ${ }^{16} \mathrm{O}+{ }^{16} \mathrm{O}$ [SLy4d], where the values are plotted without connecting lines. The left and right panels show the cases without and with the spinorbit force, respectively. Red points denote the values obtained by Eq. (3), and blue points are the values obtained by Eq. (4). For the better sight, the potential calculated after the first full overlap is shown for the negative part of $R$, while $R$ is always obtained to be positive. The potential is shown for $R / 2<6 \mathrm{fm}$.

\section{Novel dissipation mechanism}

A collision between two identical ${ }^{16} \mathrm{O}$ is calculated by three-dimensional time-dependent density functional calculations with two different Skyrme parameter sets: SLy4d [11] and SkM* [12], where the initial distance and the initial relative velocity are fixed to $20 \mathrm{fm}$ and $0.1 c$ (except for the calculation shown in Fig. 1), respectively. The corresponding center-of-mass energy is equal to $40 \mathrm{MeV}$. The first approaching stage of collision is defined by the time evolution until the first minimum of $R(t)$, so that it corresponds to the time evolution until almost $50 \mathrm{fm} / \mathrm{c}$ in case of Fig. 1. It is worth paying attention to the first approaching stage, because the long-range part of nucleus-nucleus interaction is fully included in this stage.

\subsection{Wall-window type dissipation mechanism}

Concerning the dissipation due to the mean-field, the wall-window type dissipation mechanism [4], which is also known as one-body dissipation, is fundamental. Roughly speaking the wall-window type dissipation mechanism takes into account the width of the window (neck) between the two potential walls around the contact time and the interaction between nucleons and one-body potential wall (meanfield). Accordingly this type of dissipation is less dependent on the momentum and the spatial degree of freedom. In the following a novel dissipation mechanism, which should not be classified into the wall-window type dissipation mechanism, is proposed by investigating the dynamical effect of the spin-orbit force. 

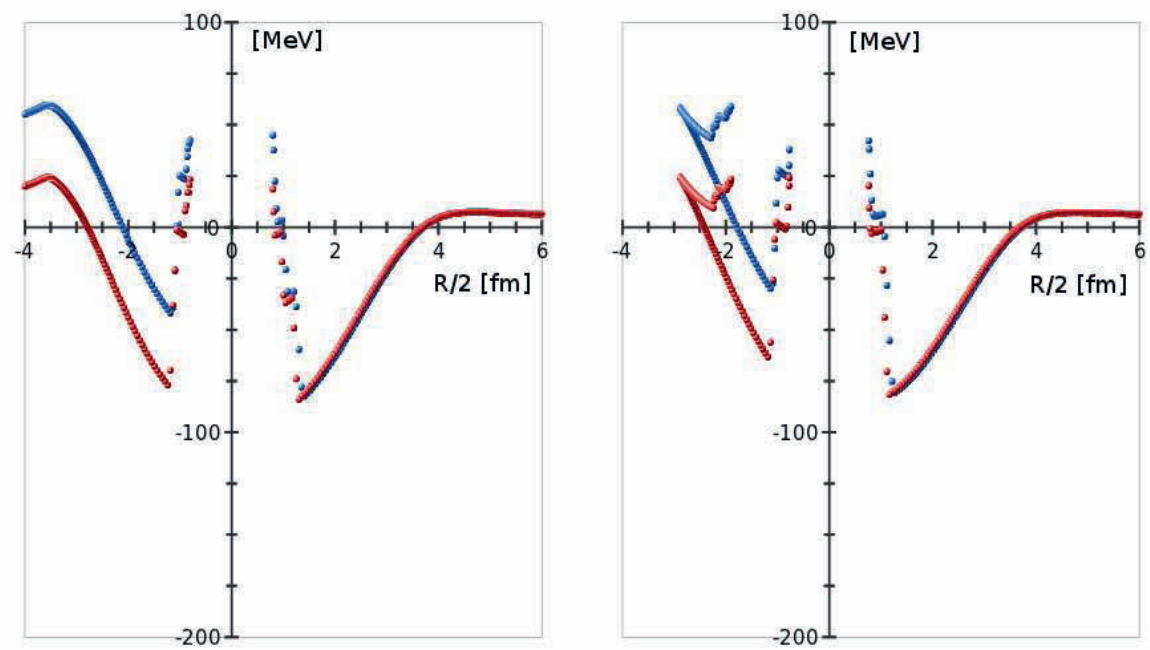

Fig. 3. Time evolutionary plot of the two values (calculated by Eqs. (3) and (4)) for ${ }^{16} \mathrm{O}+{ }^{16} \mathrm{O}[\mathrm{SkM} *$. The drawing and notation manners follow from Fig. 2.

\subsection{Spin-orbit force contribution in heavy-ion reactions}

Let us begin with the force parameter dependence of collision dynamics by comparing the results shown in Figs. 2 and 3. No qualitative difference is noticed depending on the parameter sets. Instead the coincidence of two values until the first minimum of the potential $(R>1 \mathrm{fm}$ in each panel of Figs. 2 and 3) can be confirmed independent of the parameter sets. Among other dynamical features, it is notable that the introduction of the spin-orbit force is decisive to whether fusion appears or not; the reaction results in fusion in the presence of the spin-orbit force, but non-physical passing-through takes place in the absence of the spin-orbit force (see the values at $R=-4 \mathrm{fm}$ in Figs. 2 and 3). Namely the spin-orbit force enhances the dissipation during the collision. Another important feature of the spin-orbit effect is the momentum-dependence (cf., the momentum dependence of the spin-orbit coupling). This means that the momentum-dependent dissipation arises from the spin-orbit force.

The spatial-dimension dependence of the spin-orbit force is worth mentioning, accounting for the crucial impact on final products of heavy-ion reactions. With respect to the real-time dynamics, the introduction of the spin-orbit force must be carried out in three-dimensional framework. Indeed, by comparing Fig. 1 with Fig. 1 of [2], $R(t)$-value achieves almost stationary ones around $250 \mathrm{fm} / \mathrm{c}$ only in three-dimensional calculations. Furthermore the spatial-dimension dependence is noticed to be larger than the difference between the calculation with and without beyond-the-mean-field effect. Spin-orbit effects manifesting around the edge of the density distribution appears at times $100 \mathrm{fm} / \mathrm{c}, 150 \mathrm{fm} / \mathrm{c}$ and $200 \mathrm{fm} / \mathrm{c}$ in three-dimensional framework, while they are clearly weakened in two-dimensional framework. The reason of the spatial-dimension dependence can be found in the dynamical axialsymmetry breaking due to the spin-orbit force. Indeed the axial symmetry breaking of spin distribution is shown even in central collisions (see Fig. 2 of [3] for a calculation using $\mathrm{SkM}^{*}$, and see Fig. 1 of [13] for a calculation using SLy4d). That is to say the motion perpendicular to the reaction axis 
$\mathrm{CNR} * 11$

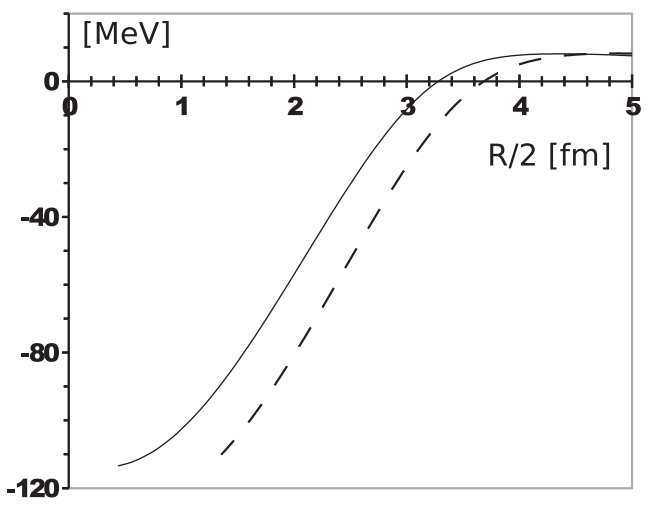

Fig. 4. Spin-orbit effect is visualized by means of nucleus-nucleus potential for ${ }^{16} \mathrm{O}+{ }^{16} \mathrm{O}[\mathrm{SLy} 4 \mathrm{~d}]$, where the initial relative velocity is equal to $0.1 c$. The solid and dashed lines show the nucleus-nucleus potential with and without the spin-orbit force, respectively.

is indispensable to take into account the spin-orbit effect. This type of dissipation depends on the momentum and the spatial degree of freedom, so that it is definitely different from the wall-window type dissipation.

As is seen in Figs 2 and 3, the two values obtained by Eqs. (3) and (4) are equal until the first minimum of the potential ( $R>1 \mathrm{fm}$ in each panel of Figs. 2 and 3$)$. As for this part the curve obtained by Eq. (3) can be regarded as the nucleus-nucleus potential. Figure 4 demonstrates the nucleus-nucleus potential for ${ }^{16} \mathrm{O}+{ }^{16} \mathrm{O}$ with and without the spin-orbit force. The spin-orbit force reduces the attraction during the first approaching stage, and the deceleration of colliding nuclei follows. In addition the introduction of the spin-orbit force brings about the changing of the entire structure of the nucleusnucleus potential. For instance it can be seen in the difference of the short-range core (Figs 2 and $3)$.

\section{Summary}

A procedure of obtaining nucleus-nucleus potentials from time-dependent density functional calculations has been presented. The role of the spin-orbit force in heavy-ion collision dynamics can be investigated using the obtained nucleus-nucleus potential. The spin-orbit force, which depends on the momentum and spatial degree of freedom, results in the large dissipation. In particular threedimensional dimensional spatial degree of freedom is necessary for the spin-orbit force to be fully operational. Consequently the spin-orbit force has been shown to bring about new type of dissipation, which is not classified into the wall-window type dissipation.

This work was supported by the Helmholtz alliance HA216/EMMI. The author thanks to Profs. H. Feldmeier, J. A. Maruhn, and T. Otsuka.

\section{References}

1. M. G. Mayer and J. H. D. Jensen, Elementary Theory of Nuclear Shell Structure, Willey, New York, 1955.

2. M. Tohyama and A. S. Umar, Phys. Rev. C 65 (2011) 037601.

3. Y. Iwata and J. A. Maruhn, Phys. Rev. C 84 (2011) 014616. 
4. W. J. Swiatecki, Phys. Scripta 24113 (1981); J. Randrup and W. J. Swiatecki, Nucl. Phys. A429 105 (1984).

5. S. E. Koonin, Prog. Part. Nucl. Phys. 4283 (1980).

6. D. Lacroix, arXiv:nucl-th/0202063.

7. K. Washiyama and D. Lacroix, Phys. Rev. C 78, 024610 (2008).

8. K. Washiyama, D. Lacroix, and S. Ayik, Phys. Rev. C79, 024609 (2009).

9. N. Loebl, J. A. Maruhn, and P.-G. Reinhard Phys. Rev. C 84 (2011) 034608.

10. Y. Iwata, H. Feldmeier, and J. A. Maruhn, to appear in AIP conf. Proc. (FINUSTAR 3).

11. K. -H. Kim, T. Otsuka, and P. Bonche, J. Phys. G 23 (1997) 1267.

12. J. Bartel, P. Quentin, M. Brack, C. Guet, and H. B. Hakansson, Nucl. Phys. A 386 (1982) 79.

13. Y. Iwata, and J. A. Maruhn, J. Phys. Conf. Ser. 295 (2011) 012126. 